\title{
Variable Fractionation of Solar Energetic Particles According to First Ionization Potential
}

\author{
R. A. Mewaldt ${ }^{1}$, C. M. S. Cohen ${ }^{1}$, R. A. Leske ${ }^{1}$, E. R. Christian ${ }^{2}$, \\ A. C. Cummings ${ }^{1}$, P. L. Slocum ${ }^{3}$, E. C. Stone ${ }^{1}$, T. T. von Rosenvinge ${ }^{2}$, \\ and M. E. Wiedenbeck ${ }^{3}$ \\ ${ }^{\prime}$ California Institute of Technology, Pasadena, CA 91125 \\ ${ }^{2}$ NASA/Goddard Space Flight Center, Greenbelt, MD 20771 \\ ${ }^{3}$ Jet Propulsion Laboratory, Pasadena, CA 91109
}

\begin{abstract}
The average composition of solar energetic particles (SEPs), like the solar corona, is known to be depleted in elements with first ionization potential (FIP) more than $\sim 10 \mathrm{eV}$ by a factor of approximately four. We examine evidence for event to event variations in the FIP-related fractionation of SEPs, following up a 1994 study by Garrard and Stone. In a survey of 46 SEP events from 1974 to 1999 the deduced FIP-fractionation varies by a factor of $\sim 2$ from event to event, with no apparent relation to charge-to-mass dependent fractionation patterns in these same events. These results are compared to similar variations observed in the solar wind.
\end{abstract}

\section{INTRODUCTION}

Variations in the elemental composition of solar energetic particles (SEPs) from event to event are usually ascribed to acceleration and transport processes that depend on the charge-to-mass $(\mathrm{Q} / \mathrm{M})$ ratio of the particles (1). In addition, it is well-known that the abundances of elements with first ionization potential (FIP) $>10 \mathrm{eV}$ are depleted in SEPs by a factor of $\sim 4$ [see, e.g., (1)]. Indeed, it was this observation that first led to the realization that the solar corona and solar wind are depleted in high-FIP elements when compared to the photosphere $(2,1,3)$. The depletion of high-FIP elements in the corona is viewed as evidence for ion-neutral separation processes that allow ionized species to be transported more efficiently from the photosphere to the corona [see, e.g., (4) and references therein]. In some models the relevant parameter is the "first ionization time" (FIT) rather than FIP [e.g., (5)].

In a 1994 paper, Garrard and Stone (6) presented evidence that the degree of FIP-fractionation in SEPs (the FIP depletion factor) varies by a factor of 2 or more from event to event, based on observations by Voyager, IMP-7\& 8 and Galileo from 1974 to 1989. Williams (7) also found the FIP-fractionation to vary in comparing two 1992 events. This paper re-examines evidence for event-to-event variations in the FIPfractionation of SEPs by combining 1974-1989 data with more recent SAMPEX and ACE observations.

\section{SOLAR PARTICLE DATA}

The SEP events in this study span the years from 1974 to 1999 and include elements from $\mathrm{C}$ to $\mathrm{Ni}$. Twenty-one events are from Breneman's study (8) of 5 to $\sim 45 \mathrm{MeV} /$ nucleon Voyager observations during 1977 to 1982. McGuire, von Rosenvinge and McDonald (9) reported 6.7 to $15 \mathrm{MeV} /$ nucleon measurements for fifteen events observed by IMP-7\&8 from 1974 to 1981 . We include the nine events not measured by Breneman, in one case substituting higher-precision ISEE-3 data (10). Adding three 1989 events from Galileo (11), we obtain the 33 events studied by Garrard and Stone (6). To these we add two 1992 events from SAMPEX (7), and eleven events from the SIS instrument on ACE, where the energy interval is $\sim 11$ to $\sim 40 \mathrm{MeV} /$ nucleon [see, e.g., (12)].

Ionic charge-state measurements are available for only a few of the SEP events in this study, mostly at lower energies than the composition data. Following Garrard and Stone, we use $\sim 1 \mathrm{MeV} /$ nucleon mean 


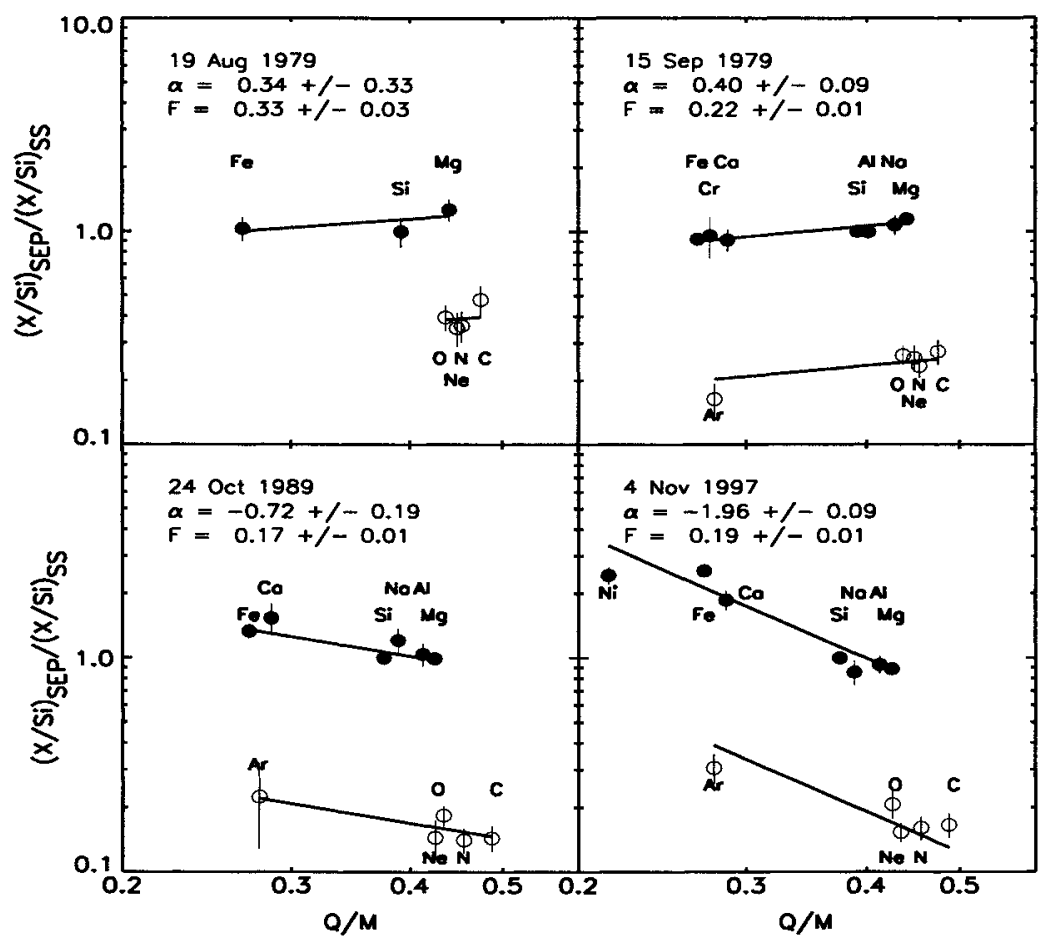

FIGURE 1. Examples of least squares fits to the normalized abundances of low-FIP (solid points) and high FIP elements (open points) in four SEP events. Best-fit vales for the Q/M fractionation index $(\alpha)$ and the FIP step factor (S) are indicated. Plotted uncertainties include uncertainties in the SEP abundances and in the tabulated solar system abundances (15).

charge states $\left\langle\mathrm{Q}_{\mathrm{Z}}>\right.$ from Luhn et al. (13), measured during 12 SEP events in 1978-1979, for Voyager, IMP-7\&8, Galileo and ISEE-3 observations. For the SAMPEX and ACE events we use $<\mathrm{Q}_{Z}>$ values measured from $\sim 15$ to $50 \mathrm{MeV} /$ nucleon by SAMPEX during two 1992 events (14).

To obtain normalized SEP abundances we define $\left.<\mathbf{J}_{Z}\right\rangle=\left(\mathrm{J}_{Z} / \mathrm{J}_{\mathrm{Si}}\right) /\left(\mathrm{P}_{Z} / \mathrm{P}_{\mathrm{Si}}\right)$ where $\mathrm{J}_{Z}$ is the fluence of element $Z$ in a given SEP event and photospheric abundances $\left(\mathrm{P}_{Z}\right)$ are taken to be Anders and Grevesse "solar system" abundances (15). We assume (1) the fractionation of element $Z$ relative to $\mathrm{Si}$ can be represented as a power-law in the ionic charge-to-mass ratio $\left(Q_{Z} / M_{Z}\right)$ multiplied by a step-function FIP fractionation factor $(F)$. Here $F=1$ for elements with $\mathrm{FIP} \leq 10 \mathrm{eV}$ and $\mathrm{F}=\mathrm{S}$ for FIP $>10 \mathrm{eV}$. Then

$$
\mathrm{J}_{Z} / \mathrm{J}_{\mathrm{Si}}=\left(\mathrm{P}_{\mathrm{Z}} / \mathrm{P}_{\mathrm{Si}}\right) \mathrm{F}(\mathrm{Z}, \mathrm{S})\left[\left(\mathrm{Q}_{\mathrm{Z}} / \mathrm{M}_{\mathrm{Z}}\right) /\left(\mathrm{Q}_{\mathrm{Si}} / \mathrm{M}_{\mathrm{Si}}\right)\right]^{\alpha} \text {. }
$$

The values of $S$ and $\alpha$ in a given SEP event are determined by a simultaneous least-squares fit to the available measured abundances (ranging from 7 to 12 species), assuming the same $\alpha$ and $S$ for all species. Sulfur (FIP $=10.4 \mathrm{eV}$ ) is not included because its SEP abundance often corresponds to intermediate values of $S(8)$. In the examples shown in Figure 1 note that both Fe-rich $(\alpha<1)$ and Fe-poor $(\alpha>1)$ events are included and that the fitted $S$ values range from $\sim 0.17$ to $\sim 0.33$.

\section{FIP-FRACTIONATION RESULTS}

The scatter-plot of $\alpha$ and $S$ values in Figure 2 indicates very little (if any) correlation between $\alpha$ and $S$, with a broad range of $\alpha$ values for both Fe-rich and Fe-poor events. It is perhaps not surprising that the fitted values of these parameters depend greatly on a few key elements with the smallest statistical uncertainties. For example, the best-fit values of $S$ are highly correlated with the measured $\mathrm{Mg} / \mathrm{O}$ ratio (see Figure 3) because the measured charge-to-mass ratios of these two abundant elements happen to be almost identical. We find $\mathrm{Q}_{\mathrm{Mg}} / \mathrm{M}_{\mathrm{Mg}}=0.440 \pm .003$ and $\mathrm{Q}_{\mathrm{O}} / \mathrm{M}_{\mathrm{O}}$ $=0.437 \pm .001$, with $\sim 3 \%$ average variation for the 12 SEP events measured by Luhn et al. (13).

Similarly, as shown in Figure 4, there is a very good correlation of $\alpha$ with the $\mathrm{Fe} / \mathrm{Si}$ ratio in a given event because these two abundant low-FIP species have a large difference in $\mathrm{Q} / \mathrm{M}\left[\mathrm{Q}_{\mathrm{Fe}} / \mathrm{M}_{\mathrm{Fe}}=0.267\right.$ and $\mathrm{Q}_{\mathrm{Si}} / \mathrm{M}_{\mathrm{Si}}=0.391$ using $\left\langle\mathrm{Q}_{\mathrm{Z}}\right\rangle$ from (13)]. The measured $\mathrm{Mg} / \mathrm{O}$ and $\mathrm{Fe} / \mathrm{Si}$ ratios therefore provide good proxies for $\mathrm{S}$ and $\alpha$. A scatter-plot of $\mathrm{S}$ and $\mathrm{Fe} / \mathrm{Si}$ is shown in Figure 5. There is no apparent correlation except possibly for very Fe-rich events. Note that event-toevent variations in $S$ cannot be due to uncertainties in the photospheric abundances. 


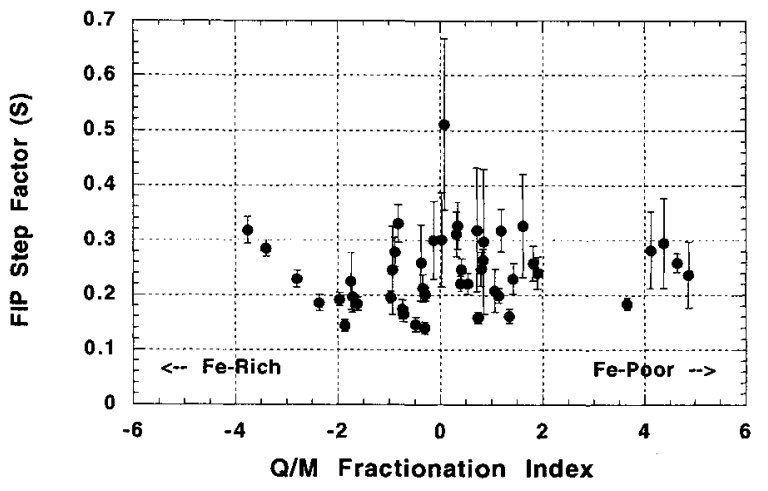

FIGURE 2. Scatter-plot of the $\mathrm{Q} / \mathrm{M}$-fractionation index $(\alpha)$ and the FIP fractionation step (S) for 46 SEP events.

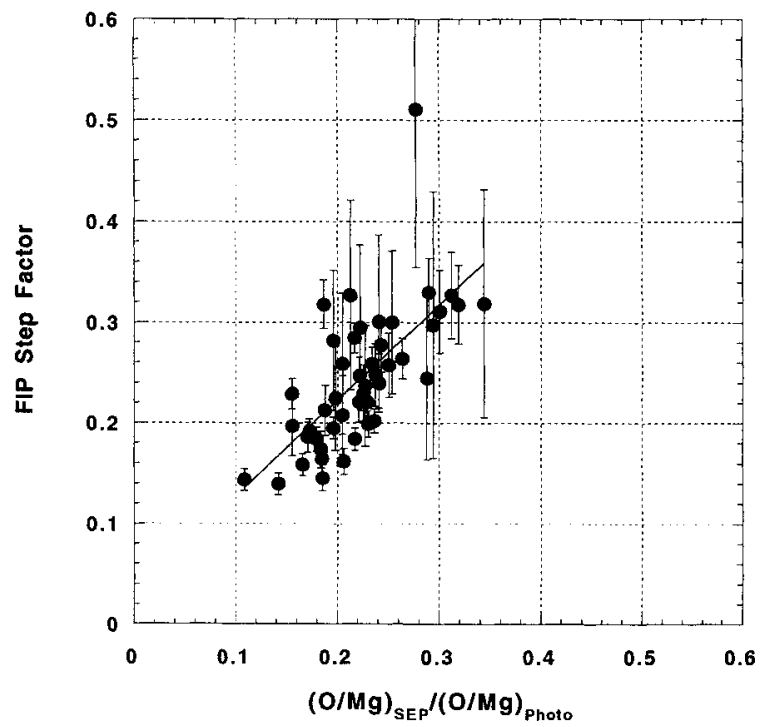

FIGURE 3. Comparison of the FIP step factor (S) with the $\mathrm{Mg} / \mathrm{O}$ ratio measured in the same event.

A potential weakness in this approach is that in 44 of the 46 events (all but the SAMPEX events) it is necessary to assume that the $\left\langle\mathrm{Q}_{\mathrm{Z}}\right\rangle$ values do not vary significantly from event to event or with energy. However, there is now evidence that $\left\langle\mathrm{Q}_{\mathrm{Z}}\right\rangle$ values in some SEP events increase with increasing energy (16, $17,18)$. Variations in $\left\langle\mathrm{Q}_{Z}\right\rangle$ will not affect the determination of $S$ in events with little $Q / M$ fractionation (small values of $\alpha$ ). However, in very $\mathrm{Fe}$-rich or very $\mathrm{Fe}$-poor events the best-fit value of $\mathrm{S}$ can be affected if the $\mathrm{Q} / \mathrm{M}$ values are considerably different than assumed. To assess this sensitivity we refit all events with two extreme assumptions: $\left\langle\mathrm{Q}_{\mathrm{Z}}\right\rangle$ characteristic of (a) $1 \mathrm{MK}$ and (b) $4 \mathrm{MK}$, based on $<\mathrm{Q}_{\mathrm{Z}}>$ calculations $(19,20)$. In these tests the best-fit values of $\alpha$ varied considerably, as expected. However, for events with $0.5 \leq<\mathrm{Fe}>\leq 2$, only one of the values of $S$ shifted by more than $\pm 15 \%$, and there was no significant difference in the distribution of S.

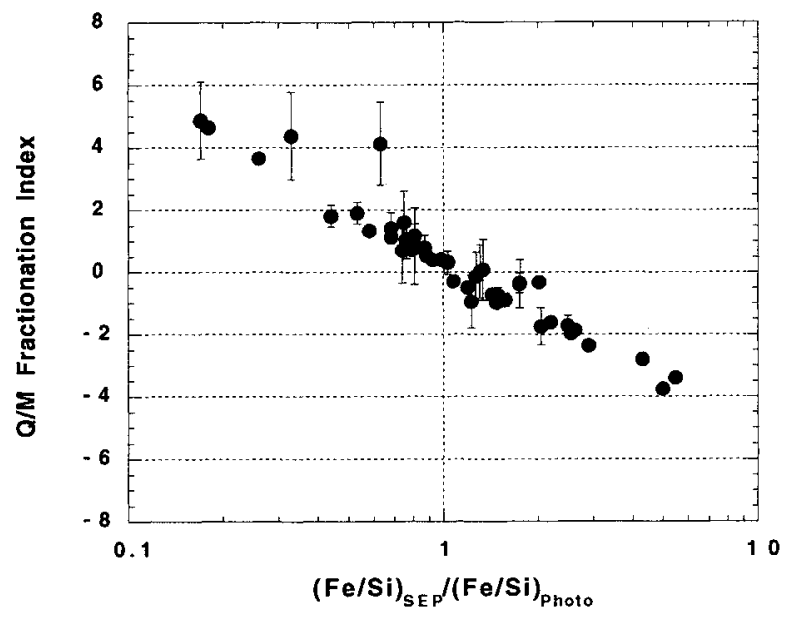

FIGURE 4. Comparison of the best-fit $\mathrm{Q} / \mathrm{M}$ fraction indices $(\alpha)$ with the $\mathrm{Fe} / \mathrm{Si}$ ratios in the same SEP events.

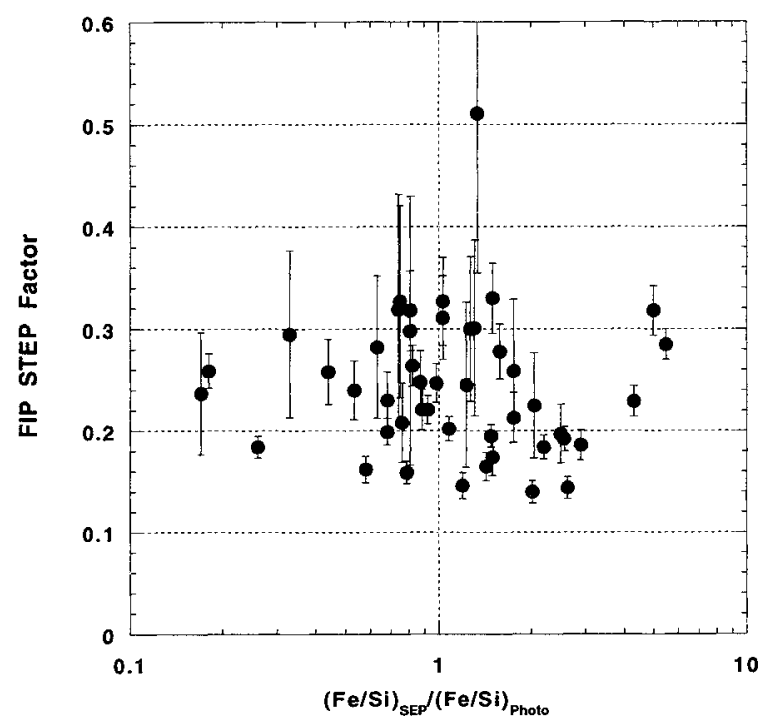

FIGURE 5. Comparison of the best-fit FIP step factors and the measured $\mathrm{Fe} / \mathrm{Si}$ ratios for all $46 \mathrm{SEP}$ events.

In order to minimize the possibility that uncertainties in $\left\langle\mathrm{Q}_{Z}\right\rangle$ are affecting the determination of $\mathrm{S}$ we confine the subsequent discussion to events with $\langle\mathrm{Fe}\rangle|<\mathrm{Si}\rangle$ ratio ranges from 0.5 to 2 , a maximum variation of a factor of 2 from the solar system value. Figure 6 shows the distribution of $S$ values for these $31 \mathrm{SEP}$ events. The mean value is 0.254 with an rms deviation of 0.056 , in reasonable agreement with the results of Garrard and Stone (6), where the mean was $S=0.23$ with an rms deviation of 0.11 . Differences from their study appear to be due to their use of a different set of photospheric abundances, with a greater abundance of $\mathrm{Fe}$, and to our omission of very Fe-rich and Fe-poor events, which, in our opinion, provide less reliable FIP-fractionation estimates. 


\section{DISCUSSION}

The solar wind also exhibits a variable degree of FIP fractionation. In the slow solar wind the FIP fractionation factor $S$ is similar to the average SEP value of 0.25 , but the FIP effect is reduced in highspeed solar wind streams [e.g., (5)]. There are also variations in the degree of FIP fractionation determined from coronal spectroscopy studies (21).

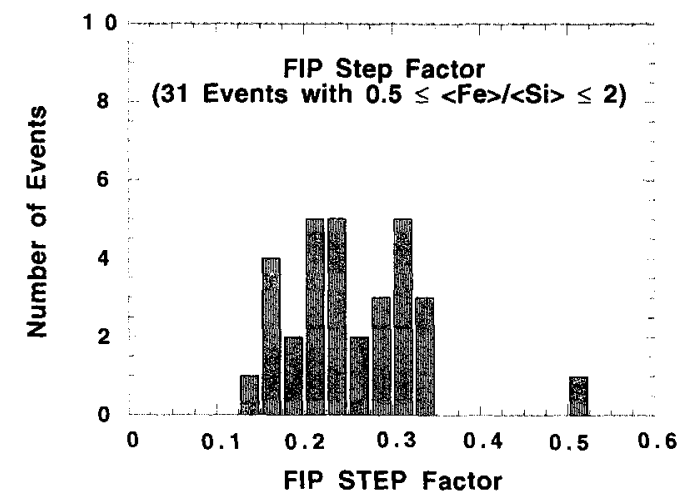

FIGURE 6. Distribution of FIP step factors (S) for the 31 SEP events with $0.5 \leq<\mathrm{Fe}>/</ \mathrm{Si}>\leq 2$.

The fact that solar wind and SEPs show similar variations in the degree of FIP fractionation suggests that these variations have a common origin. This might arise if the seed populations for both solar wind and SEPs include coronal material within which the FIP fractionation varies, or if the SEP seed population is a variable mix of fast and slow solar wind.

In summary, the results presented here confirm and extend those of Garrard and Stone (7) and Williams (8). We confirm the finding that the degree of FIP fractionation (the FIP depletion factor) varies by about a factor of two from event to event. The distribution of $\alpha$ and $\mathrm{S}$ values (Figure 2) shows no evidence for a correlation between the degree of FIP-fractionation and the degree of $\mathrm{Q} / \mathrm{M}$-dependent fractionation. It is interesting that variations in the degree of FIP fractionation of SEPs are similar to variations observed in the solar wind, suggesting that they may have a common origin.

\section{ACKNOWLEDGMENTS}

This work was supported by NASA at the California Institute of Technology (under grant NAS56912), the Jet Propulsion Laboratory, and Goddard Space Flight Center.

\section{REFERENCES}

1. Breneman, H., and Stone, E. C., Astrophys. J. Letters 299, L57-L61 (1985).

2. Mewaldt, R. A., in Proc. Conf. Ancient Sun, R. O. Pepin, J. A. Eddy, and R. B. Merrill, eds, (Pergamon, New York), 81-101 (1980).

3. Meyer, J. P., Ap. J. Suppl, 57, 151 (1985).

4. Henoux, J.-C., Space Science Reviews 85, 215-226 (1998).

5. Geiss, J., Space Science Reviews 85, 241-252 (1998).

6. Garrard, T. L., and Stone, E. C., Adv. Space Res. 14, (10) 589-598 (1994).

7. Williams, D. L. PhD Thesis, Caltech (1996).

8. Breneman, H. H, PhD Thesis, Caltech (1985).

9. McGuire, R. E., von Rosenvinge, T. T., and McDonald, F. B., Ap. J. 301, 938-961 (1986).

10. Mewaldt, R. A., Spalding, J. D., and Stone, E. C., Ap. J. 280, 892-901 (1984).

11. Garrard, T. L. and Stone, E. C., "Heavy Ions in the October 1989 Solar Flares Observed on the Galileo Spacecraft", Proceedings of the 22nd Internat. Cosmic Ray Conf. (Dublin) 3, 331-334 (1991).

12. Cohen, C. M. S., et al., Geophys. Res. Letters 26, $2697-$ 2700 (1999).

13. Luhn, A., et al. Adv. Space Research 4, 161 (1984).

14. Leske, R. A., et al., Ap. J. 452, L149-L152 (1995).

15. Anders, E., and Grevesse, N., Geochim. Cosmochim. Acta 53, 197-214 (1989).

16. Oetliker, M., et al., Ap. J. 477, 495-501 (1997).

17. Mazur, J. E., et al., Geophys. Res. Letters 26, 173-176 (1999).

18. Moebius. E., et al., Geophys. Res. Letters 26, 173-176 (1999).

19. Arnaud, M., and Rothenflug, R., Astron. Astrophys. Suppl. Ser. 60, 425-457 (1985).

20. Arnaud, M., and Raymond, J., Astrophys. J. 398, 394406 (1992)

21. Feldman, U., Space Science Reviews, 85, 227-240 (1998). 